\title{
New Reassortant H5N6 Highly Pathogenic Avian Influenza Viruses in Southern China, 2014
}

\author{
Peirong Jiao ${ }^{1,2,3,4 t}$, Jin Cui 1,2,3,4t, Yafen Song ${ }^{1,2,3,4}$, Hui Song ${ }^{1,2,3,4}$, Zhishan Zhao ${ }^{1,5}$, \\ Siyu Wu ${ }^{1,2,3,4}$, Nannan $Q u^{1,2,3,4}$, Nianchen Wang ${ }^{1,2,3,4}$, Guowen Ouyang ${ }^{1,2,3,4}$ and \\ Ming Liao ${ }^{1,2,3,4 *}$
}

1 National and Regional Joint Engineering Laboratory for Medicament of Zoonosis Prevention and Control, Guangzhou, China, ${ }^{2}$ Key Laboratory of Animal Vaccine Development, Ministry of Agriculture, Guangzhou, China, ${ }^{3}$ Key Laboratory of Zoonosis Prevention and Control of Guangdong, Guangzhou, China, ${ }^{4}$ College of Veterinary Medicine, South China Agricultural University, Guangzhou, China, ${ }^{5}$ College of Life Science, South China Agricultural University, Guangzhou, China

OPEN ACCESS

Edited by:

Hirofumi Akari

Kyoto University, Japan

Reviewed by:

Shailesh D. Pawar,

National Institute of Virology, India

Guillermo Tellez,

University of Arkansas, USA

*Correspondence:

Ming Liao

mliao@scau.edu.cn

tThese authors have contributed equally to this work

Specialty section: This article was submitted to Virology,

a section of the journal Frontiers in Microbiology

Received: 30 March 2016 Accepted: 04 May 2016

Published: 19 May 2016

Citation:

Jiao P, Cui J, Song Y, Song $H$,

Zhao Z, WUS, QuN, Wang N, Ouyang $G$ and Liao M (2016) New Reassortant H5N6 Highly Pathogenic Avian Influenza Viruses in Southern China, 2014. Front. Microbiol. 7:754

doi: 10.3389/fmicb.2016.00754
New reassortant H5N6 highly pathogenic avian influenza viruses (AIVs) were isolated from apparently healthy domestic ducks in Southern China in 2014. Our results show that the viruses grew efficiently in eggs and replicated systemically in chickens. They were completely lethal in chicken (100\% mortality), and the mean death time was 6 to 7 days post-inoculation. The viruses could transmit in chickens by naïve contact. BLAST analysis revealed that their HA gene was most closely related to A/wild duck/Shangdong/628/2011 (H5N1), and their NA genes were most closely related to A/swine/Guangdong/K6/2010 (H6N6). The other genes had the highest identity with A/wild duck/Fujian/1/2011(H5N1). The results of phylogenetic analysis showed that their $\mathrm{HA}$ genes clustered into clade 2.3.4.4 of the H5N1 viruses and all genes derived from $\mathrm{H} 5$ were Mix-like or H6-like viruses. Thus, the new H5N6 viruses were reassortmented of $\mathrm{H} 5 \mathrm{~N} 1$ and $\mathrm{H} 6 \mathrm{~N} 6$ virus. Therefore, the circulation of the new H5N6 AlVs may become a threat to poultry and human health.

Keywords: H5N6, highly pathogenic avian influenza virus, ducks, evolution, pathogenicity

\section{INTRODUCTION}

Waterfowl are a natural reservoir of avian influenza A viruses (Kawaoka et al., 1988). Most subtypes of avian influenza A viruses have been found in aquatic birds (Webster et al., 1992). Southern China is one of the main areas for farming of domestic waterfowl where they often share water with wild aquatic birds. Most avian influenza viruses (AIVs) do not cause disease in humans. However, some are zoonotic meaning that they can infect humans and cause disease The most well-known example is the avian influenza subtype H5N1 viruses that are currently circulating in poultry in parts of Asia and northeast Africa. These have caused human disease and deaths since 1997. In recent years, a variety of novel avian viruses have been detected in poultry in Guangdong Province. An increasing number of overwhelming viral infections have aroused public awareness of zoonotic avian influenza transmission. Notably, Zhejiang, Hubei, and Guangdong had H5N6 avian influenza in farms in 2014 followed by Hunan and Jiangsu in 2015 (World Organization for Animal Health, 2015). Whenever AIVs are circulating in poultry, sporadic infections and small clusters of human cases are possible in people exposed to infected poultry 
TABLE 1 | Replication and lethality in chickens of the H5N6 viruses after intranasal infection ${ }^{\mathrm{a}}$.

\begin{tabular}{|c|c|c|c|c|c|c|c|c|}
\hline \multirow[t]{2}{*}{ Strains } & \multirow[t]{2}{*}{ Titer $\left(\log _{10} \mathrm{EID}_{50}\right)$} & \multicolumn{6}{|c|}{ Virus replication on 3 days post-inoculation (DPI; $\left.\log _{10} \mathrm{EID}_{50} / 0.1 \mathrm{ml}\right)^{\mathrm{b}}$ in } & \multirow{2}{*}{$\begin{array}{c}\text { Manifestations of chickens } \\
\text { No. D/S/total }{ }^{c}\end{array}$} \\
\hline & & Heart & Liver & Spleen & Lung & Kidney & Brain & \\
\hline 018 & 8.63 & $4.00 \pm 0.00$ & $5.92 \pm 0.51$ & $5.25 \pm 0.29$ & $6.75 \pm 0.87$ & $6.58 \pm 0.68$ & $5.25 \pm 0.13$ & $10 / 10 / 10$ \\
\hline 021 & 8.54 & $3.92 \pm 0.01$ & $5.5 \pm 0.67$ & $5.08 \pm 0.26$ & $6.17 \pm 0.35$ & $5.92 \pm 0.51$ & $5.33 \pm 0.22$ & $10 / 10 / 10$ \\
\hline
\end{tabular}

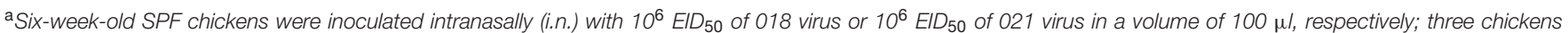

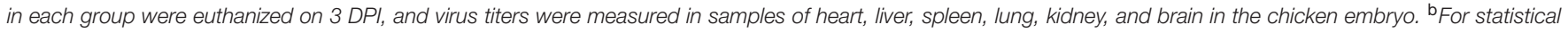

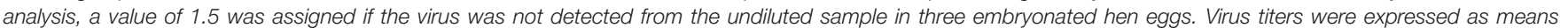

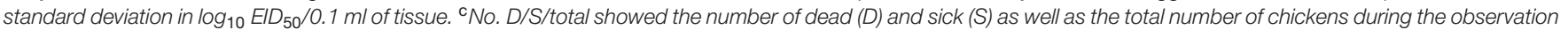
period. Birds that showed disease signs such as depression and ruffled feathers, but recovered at the end of the observation, were considered to be sick animals.

or contaminated environments. Therefore, new H5N6 viruses should be monitored for their impact on animal and human health.

\section{MATERIALS AND METHODS}

\section{Viruses}

The H5N6 HPAI viruses A/duck/Guangzhou/018/2014 (018) and A/duck/Guangzhou/021/2014 (021) were isolated from swabs of ducks in a live bird market in Guangdong, China by the Key Laboratory of Animal Disease Control and Prevention, Ministry of Agriculture of P.R. China. They were purified and propagated in 9- to 10-day-old specific-pathogen-free (SPF) embryonated hen eggs. Values of $50 \%$ egg infective doses $\left(\mathrm{EID}_{50}\right)$ were calculated by the Reed-Muench method. All experiments were carried out in ABSL-3 facilities.

\section{Sequence Analysis}

The viral genes used in this study were sequenced. Viral RNA was extracted from allantoic fluid with Trizol LS Reagent (Life Technologies, Inc.) and transcribed into cDNA with SuperScript III reverse transcriptase (Invitrogen). PCR amplification was performed using fragment-specific primers that matched the conserved end sequence of the RNA fragments of the influenza virus. The PCR products were purified with the QIAquick PCR purification kit (QIAGEN) and sequenced using an automatic ABI Prism 3730 genetic analyzer (Applied Biosystems) according to the manufacturer's instructions. The DNA sequences were compiled and edited using Lasergene 7.1 (DNASTAR). A phylogenetic tree of the H5N6 influenza A viruse s was generated by the distancebased neighbor-joining method using software MEGA 5 (Sinauer Associates, Inc., Sunderland, MA, USA). The reliability of the tree was assessed by bootstrap analysis with 1000 replicates. Horizontal distances are proportional to genetic distance. The nucleotide sequences obtained in the present study are available from GenBank under the accession numbers (KX094400KX094415).

\section{Infection Study}

Six-week-old specific pathogen-free White Leghorn chickens $(n=20)$ were housed in isolator cages and divided into two groups with 10 per group. In each group, seven chickens were intranasally (i.n.) inoculated $\left(10^{6} \mathrm{EID}_{50}\right.$ in $\left.0.2 \mathrm{ml}\right)$ with one of the two viruses. Three chickens were inoculated with $0.2 \mathrm{ml}$ phosphate buffered saline (PBS) as negative controls. All chickens were observed for clinical symptoms for 14 days. Post-infection day 3 (DPI), three inoculated chickens in each group were euthanized to test for viral replication in the organs including the lung, kidney, brain, heart, spleen, and liver. All tissues were collected and titrated for viral infectivity in eggs. All experiments were carried out in ABSL-3 facilities in compliance with the biosafety committee of South China Agriculture University protocols. The chicken handling was performed in accordance with experimental animal administration and the ethics committee of South China Agriculture University guidelines.

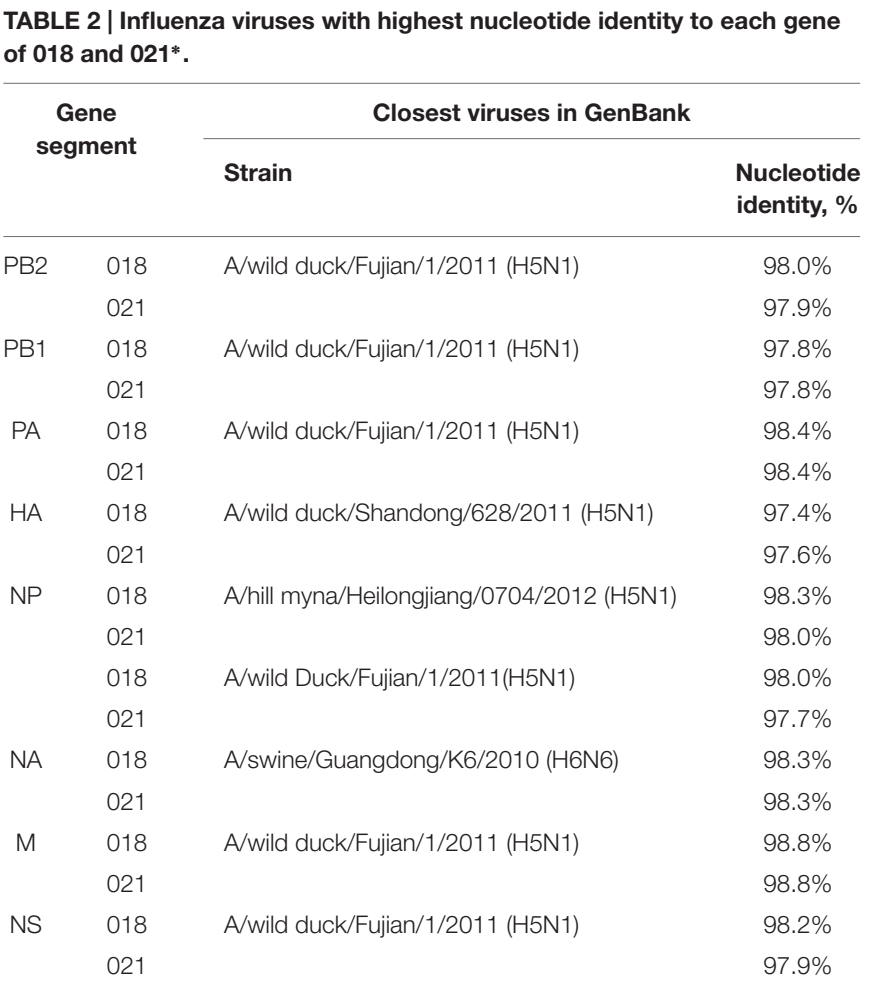

*018, A/duck/Guangzhou/018/2014(H5N6); 021, A/duck/Guangzhou/021/2014 (H5N6); PB, polybasic protein; PA, polymerase acidic protein; HA, hemagglutinin; NP, nucleocapsid protein; NA, neuraminidase; M, matrix protein; and NS, nonstructural protein. 


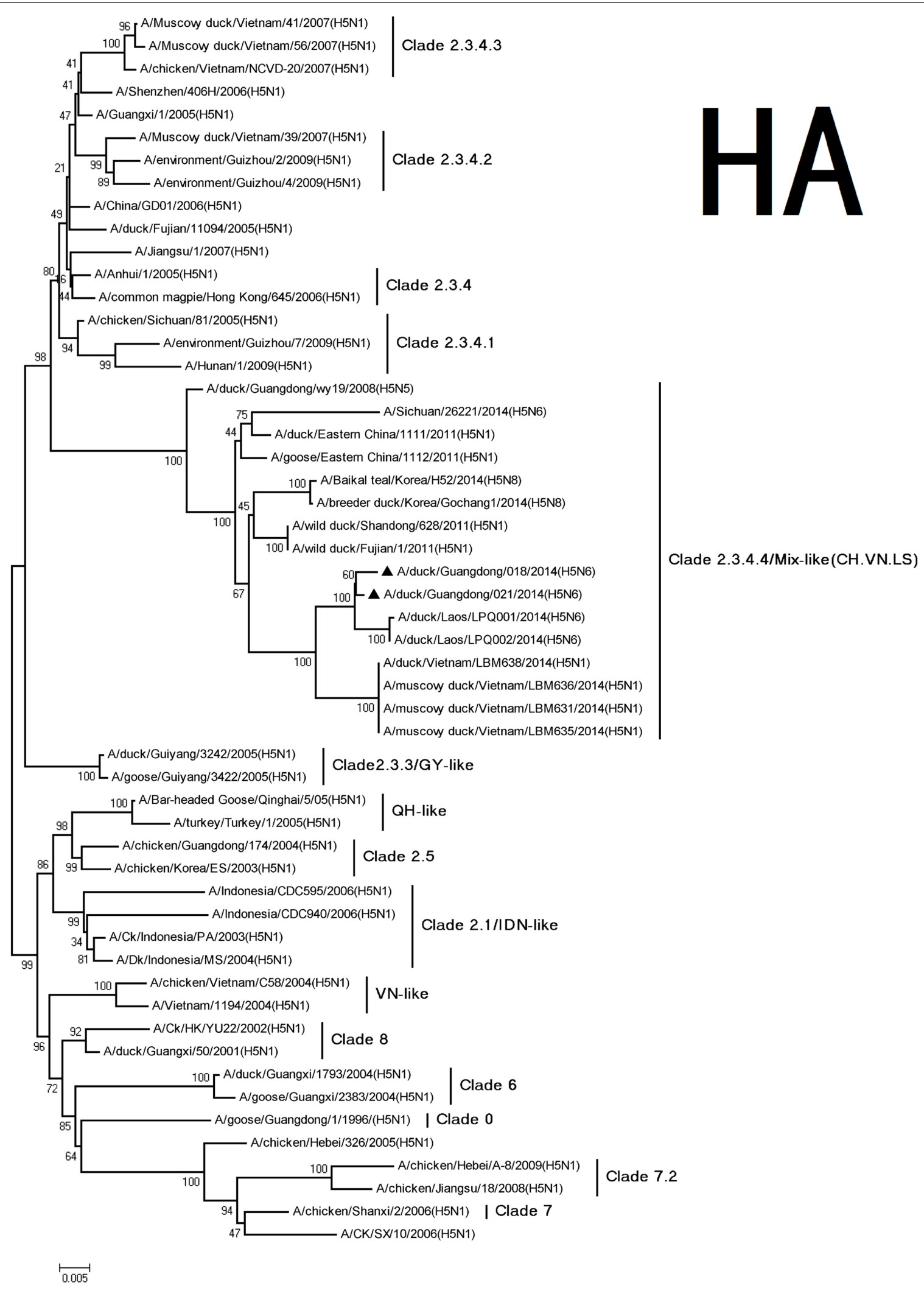

FIGURE 1 | Phylogenetic analysis of HA. The trees were constructed using the neighbor joining method with the Maximum Composite Likelihood model and MEGA version 5.2 (www.megasoftware.net/) with 1,000 bootstrap replicates based on the following sequences: HA (A): nucleotides (nt) 29 to 1732 . The triangles indicated viruses characterized in this study. Other viral sequences were downloaded from GenBank. QH, QingHai; VN, Vietnam; IDN, Indonesia; and GY, GuiYang. 


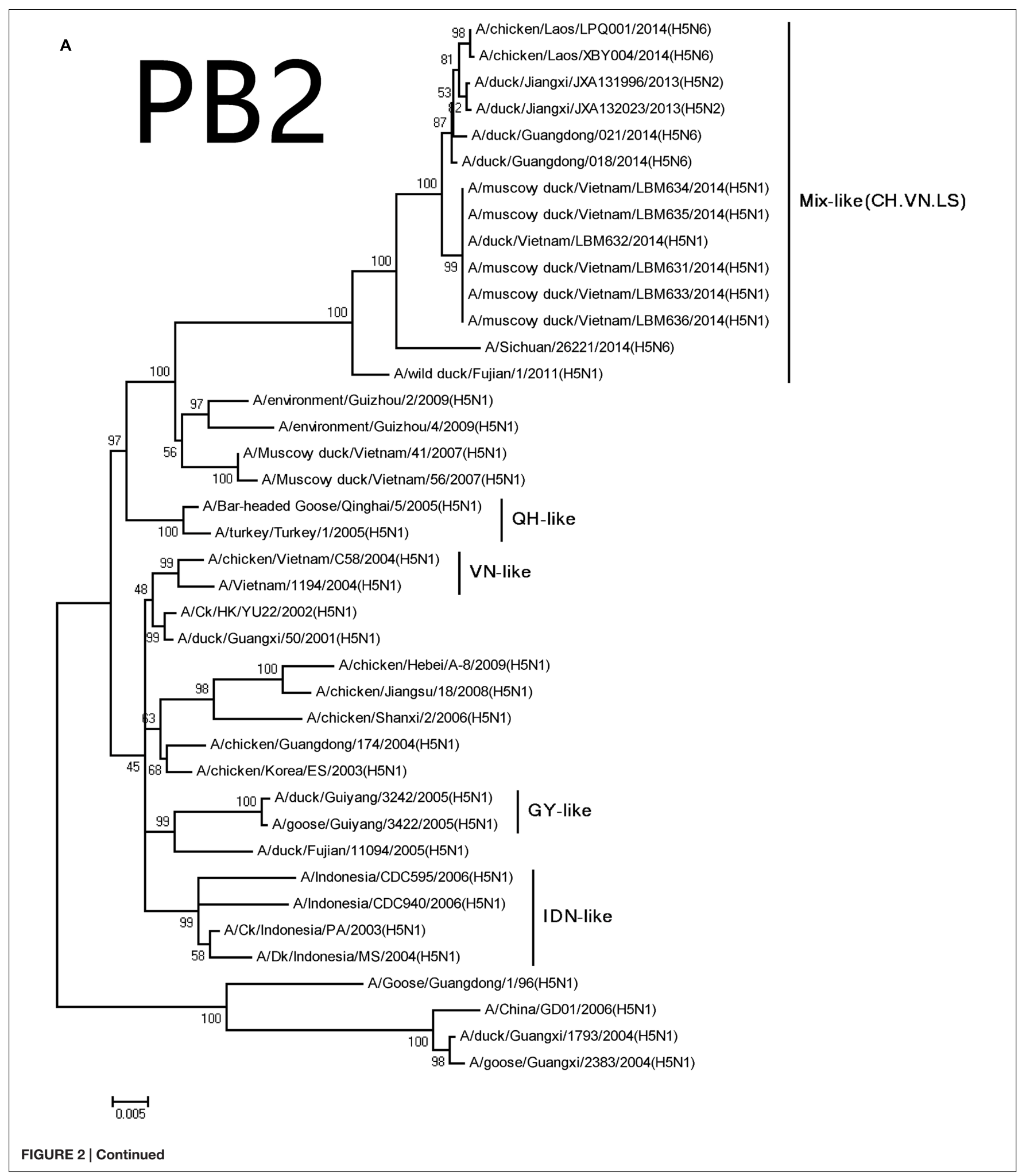

\section{RESULTS}

In early March 2014, H5N6 AIVs-A/duck/Guangzhou/018/ 2014 (018) and A/duck/Guangzhou/021/2014 (021) -were isolated from samples of ducks as described (Sun et al., 2011). The viruses grew efficiently in eggs, and both had virus titers $>8$ $\log 50 \%$ egg infectious dose $\left(\mathrm{EID}_{50}\right) / 0.1 \mathrm{~mL}$. To understand the pathogenicity of the viruses in chicken, 6-week-old SPF chickens 


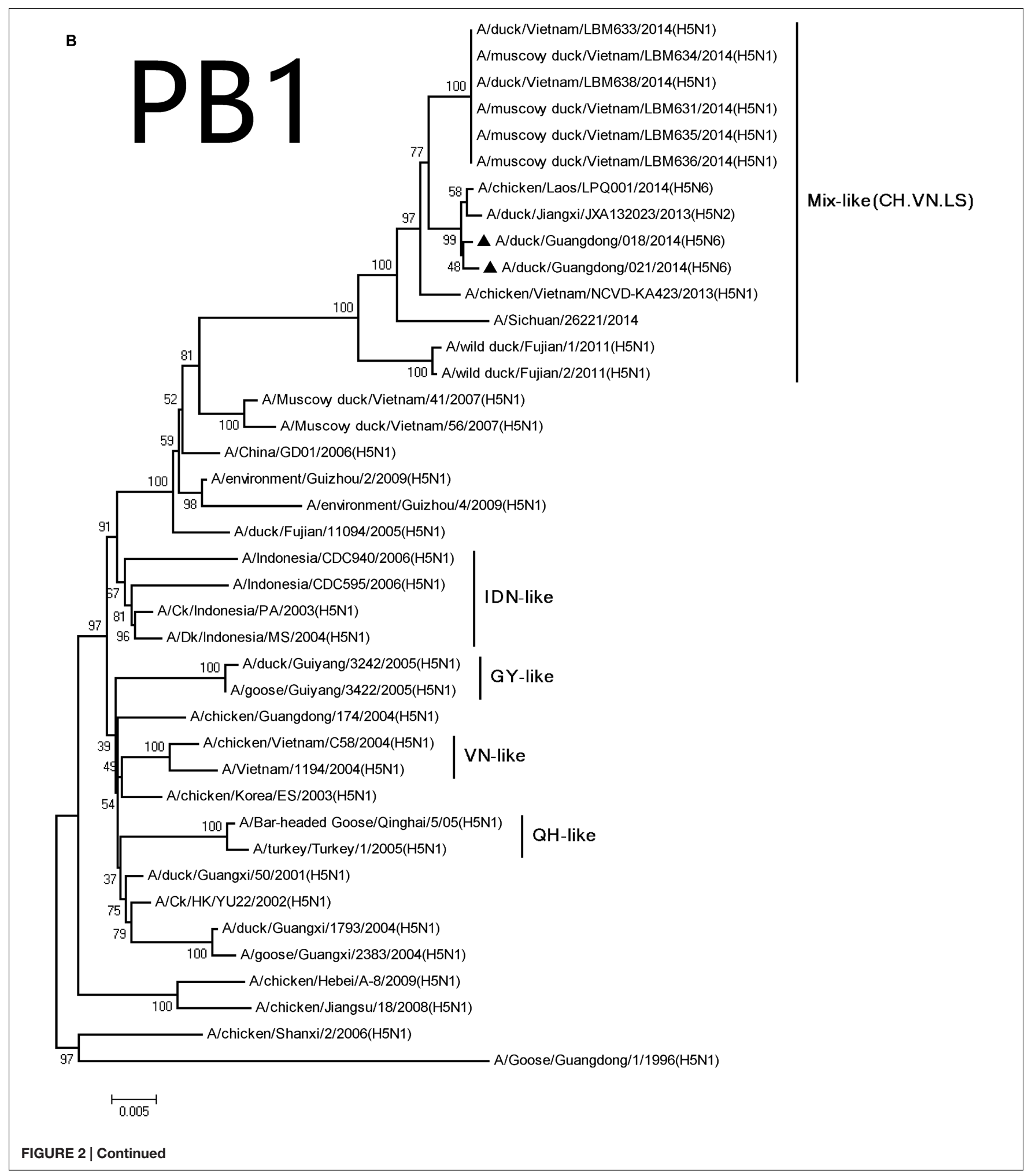

were inoculated intranasally (i.n.) with $10^{6} \mathrm{EID}_{50}$. All chickens were observed for 14 days after infection.

In this test, the infected chickens exhibited anorexia and depression at 1 DPI. All chickens of the 018 group died within
6 DPI. All chickens inoculated with 021 died within 7 DPI. Moreover, the viruses could transmit to chickens by naive contact. All naïve contact chickens housed with those inoculated with 018 virus died at 4-7 DPI. Three naïve contact chickens died 


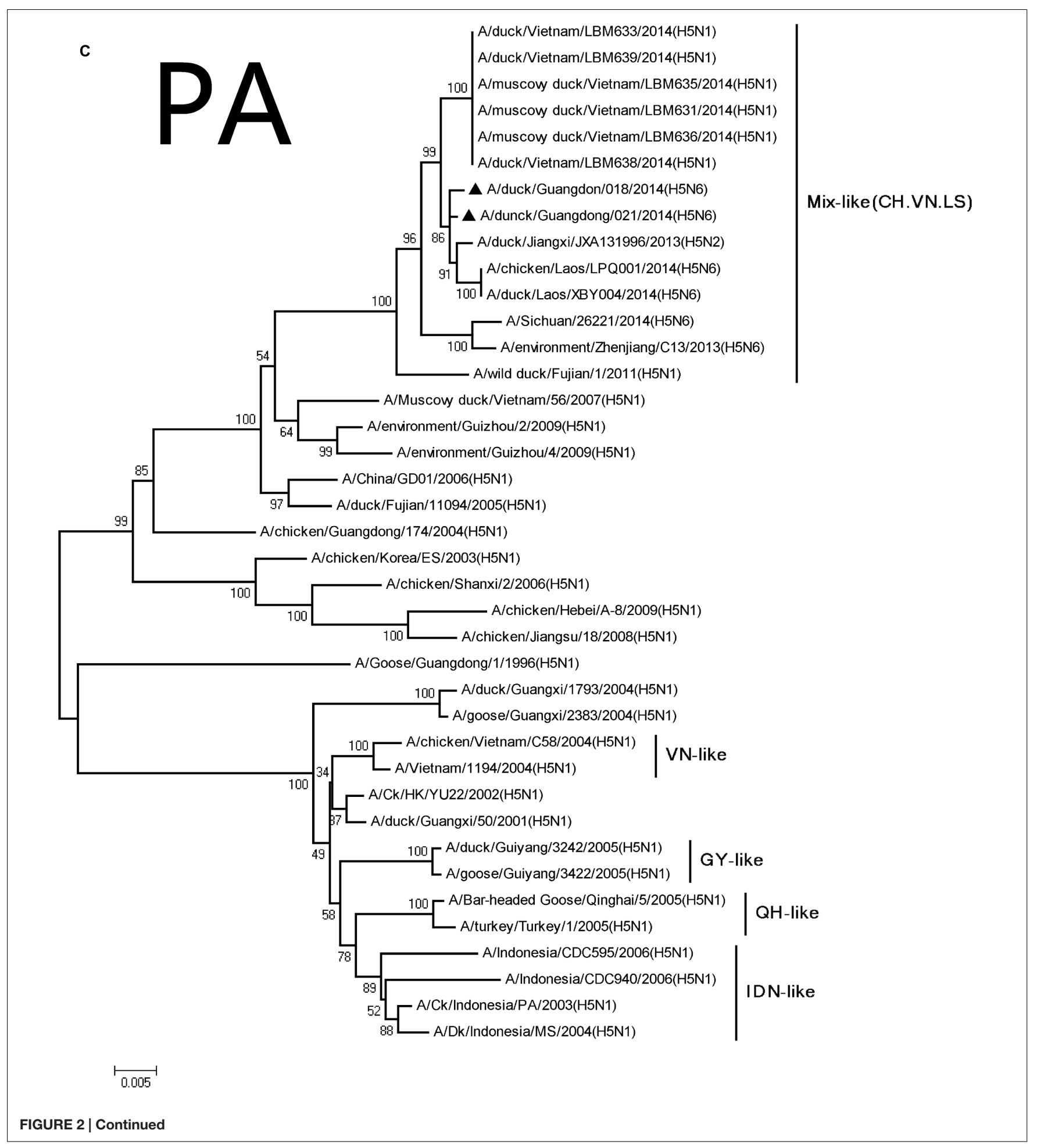

in the 021 group at 5-6 DPI. The viruses replicated systemically in chickens, and this could be detected in all the tested organs by 3 DPI including the heart, liver, spleen, lung, kidney, and brain (Table 1). The 018 virus replicated more highly in the lungs (6.75 $\left.\log _{10} \mathrm{EID}_{50}\right)$. The mean virus titers in the heart, liver, spleen, kidney, and brain were 4.00, 5.92, 5.25, 6.58, and $5.25 \log _{10} \mathrm{EID}_{50}$, respectively. The 021 virus replicated in all tested organs and the highest virus titers was detected in the chicken lung $\left(6.17 \log _{10}\right.$ $\mathrm{EID}_{50}$; Table 1).

All eight genes were sequenced to trace the origin and understand the genetic properties of the viruses. We performed phylogenetic analyses of these sequences and compared 


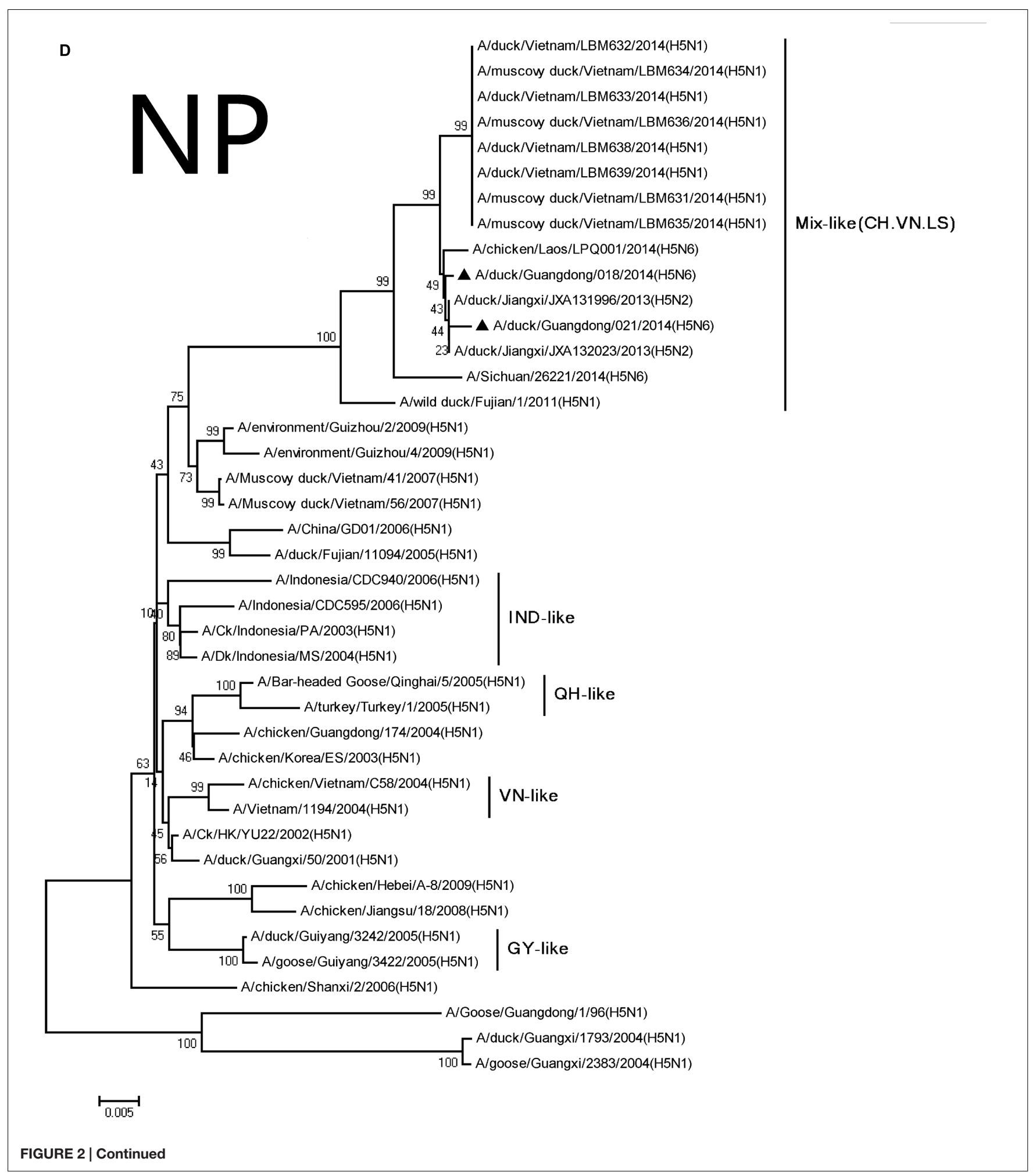

them with sequence data obtained from GenBank. BLAST ${ }^{1}$ analysis revealed that their HA genes were highly homologous with A/wild duck/Shandong/628/2011(H5N1) virus, and the

${ }^{1}$ www.ncbi.nlm.nih.gov/genomes/FLU/FLU.html nucleotide sequence homology was up to 97.4 and $97.6 \%$, respectively (Table 2).

According to antigenic characteristics by the World Health Organization/World Organization for Animal Health/Food, Agriculture Organization (WHO/OIE/FAO H5N1 Evolution 


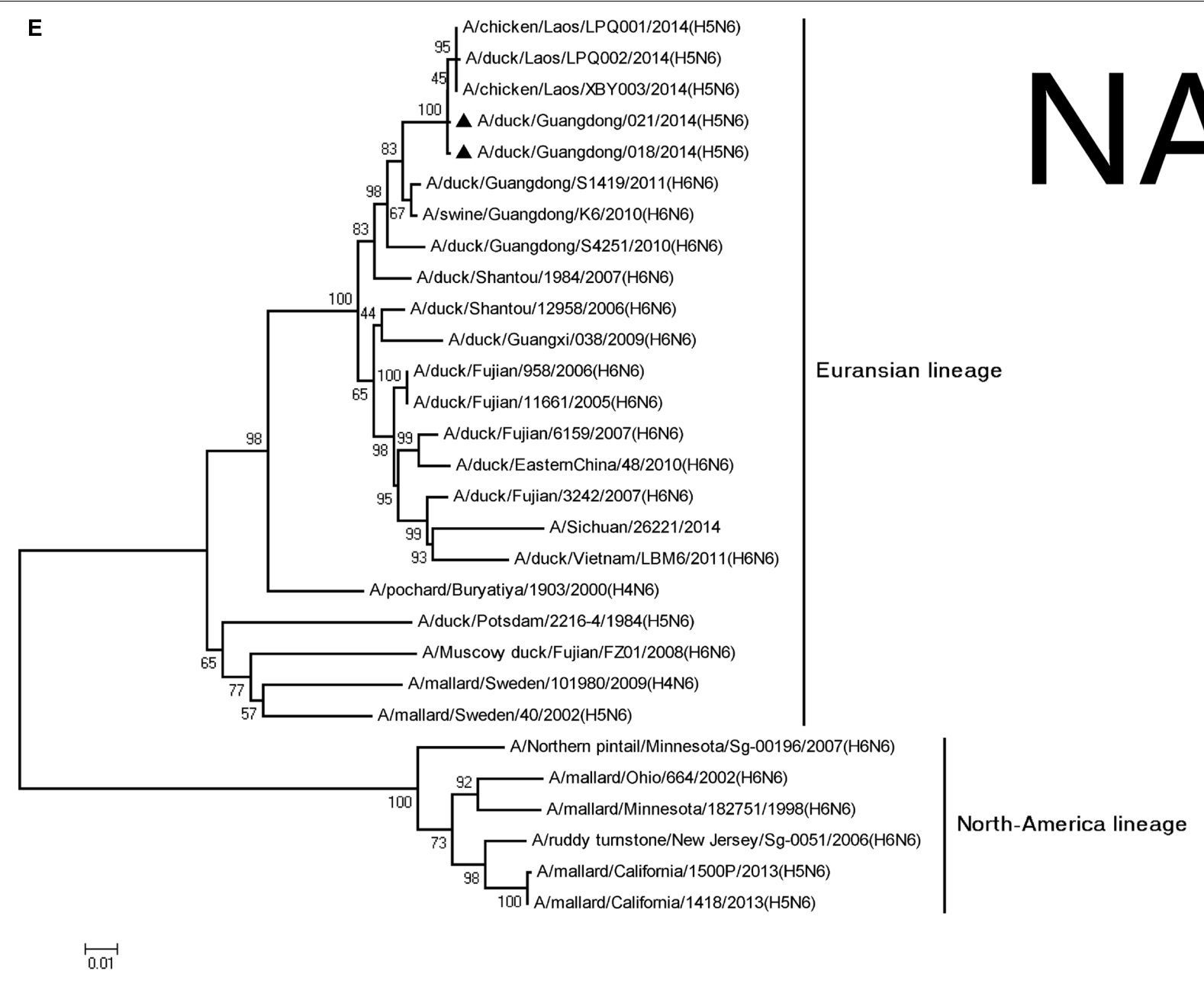

FIGURE 2 | Continued

Working Group, 2012), the HA genes of the H5N6 viruses were classified into clade 2.3.4. They could further be classified into subclade 2.3.4.4 and belong to Mix-like viruses, which include a H5N6 virus-infected human in Sichuan in 2014 (Figure 1) (Gu et al., 2013). They have a series of deduced basic amino acid sequences (PLRERRRKR/GLF) at cleavage sites of HA, which was characteristic of highly pathogenic AIVs (Nobusawa et al., 1991; Belser et al., 2009). Conservative amino acid residues within receptor binding pockets of HA (including E190, G225, Q226, and G228; H3 numbering) were present in the viruses. This indicated that they may retain affinity for the avian (sialic acid-2, 3-NeuAcGal) cell surface (Stevens et al., 2006). A previous study showed the $5227 \mathrm{~N}$ substitution accompanied by deglycosylation at residue 158 could substantially increase the affinity of HA for $\mathrm{SA} \alpha 2$, 6Gal without reducing its binding affinity for $\mathrm{SA} \alpha 2$, $3 \mathrm{Gal}$ (Yen et al., 2009). In our study, the H5N6 viruses simultaneously carried an S227R mutation in the receptorbinding pocket. The impact of this S227R variation with a changed residual polarity on receptor binding deserves further investigation.
The NA genes of the viruses had high homology with A/swine/Guangdong/K6/2010 (H6N6; NA: 018, 98.3\%; 021, 98.3\%; Table 2). We retrieved complete NA sequences from GenBank (including former H5N6 viruses from North America and Europe) to compare to the viruses containing the N6 gene. The H5N6 isolates belonged to the Eurasian lineage H6N6-like viruses (Figure 2). An 11-amino acid deletion of residues 5969 were found in the NA genes, but not in the previous H5N6 viruses.

The PB2 and PB1 genes were most closely related to those of the A/wild duck/Fujian/1/2011(H5N1) virus (PB2:018, 98.0\%; 021, 97.9\%, PB1:018, 97.8\%; 021, 97.8\%), respectively, (Table 2). The genes were clustered into Mix-like viruses, which included a H5N6 virus-infected human in Sichuan in 2014 (Figure 2). The PB2 $627 \mathrm{~K}$ allows avian-derived viral polymerases to evade a cellular inhibitor in human cells (Mehle and Doudna, 2008), but this mutation was not observed in PB2 of the new H5N6 viruses.

The PA and $\mathrm{M}$ genes nucleotide sequence homology were both 98.4 and $98.8 \%$ compared with the A/wild duck/Fujian/1/2011(H5N1) virus, respectively, (Table 2). 


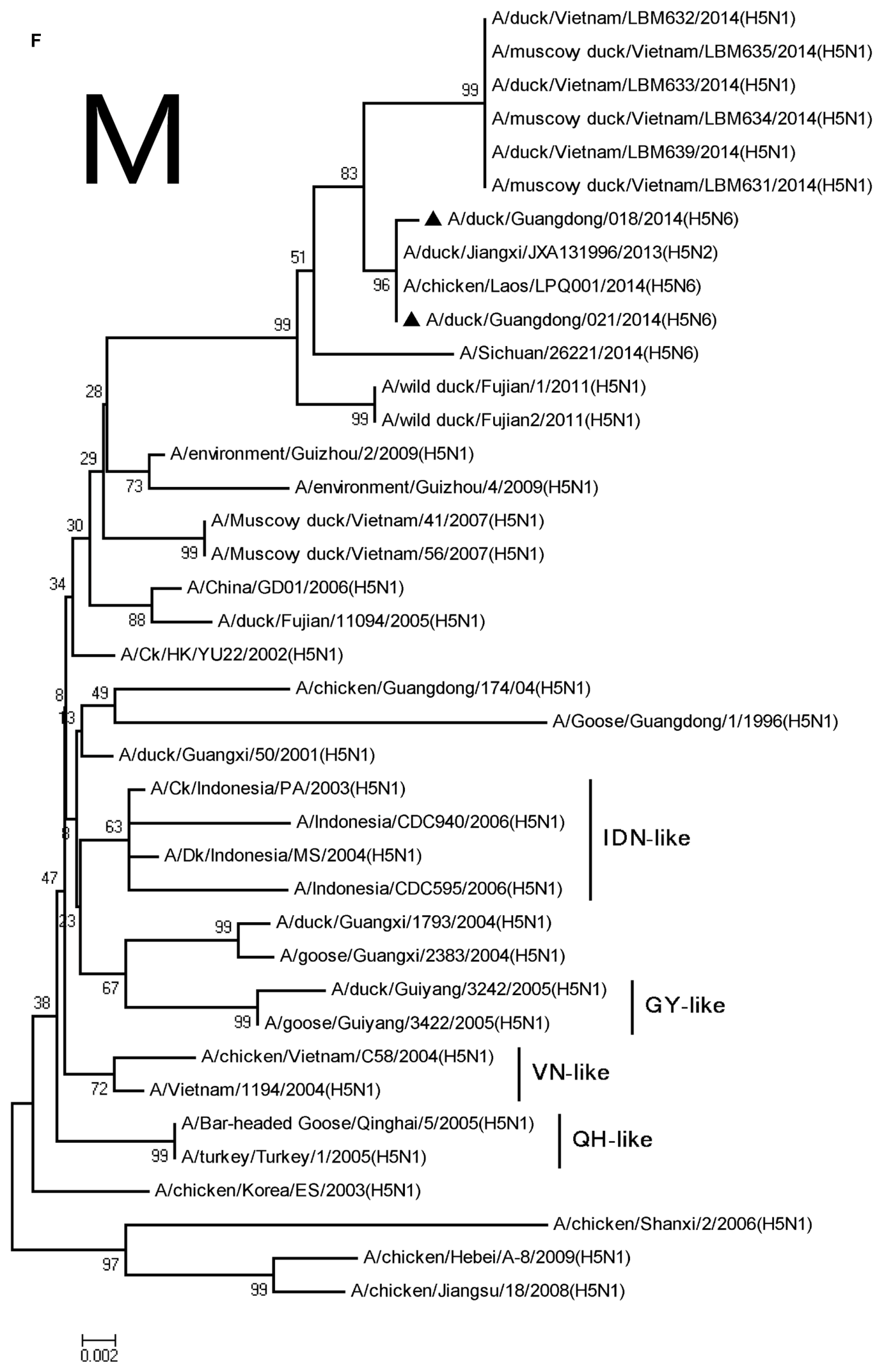

Mix-like 


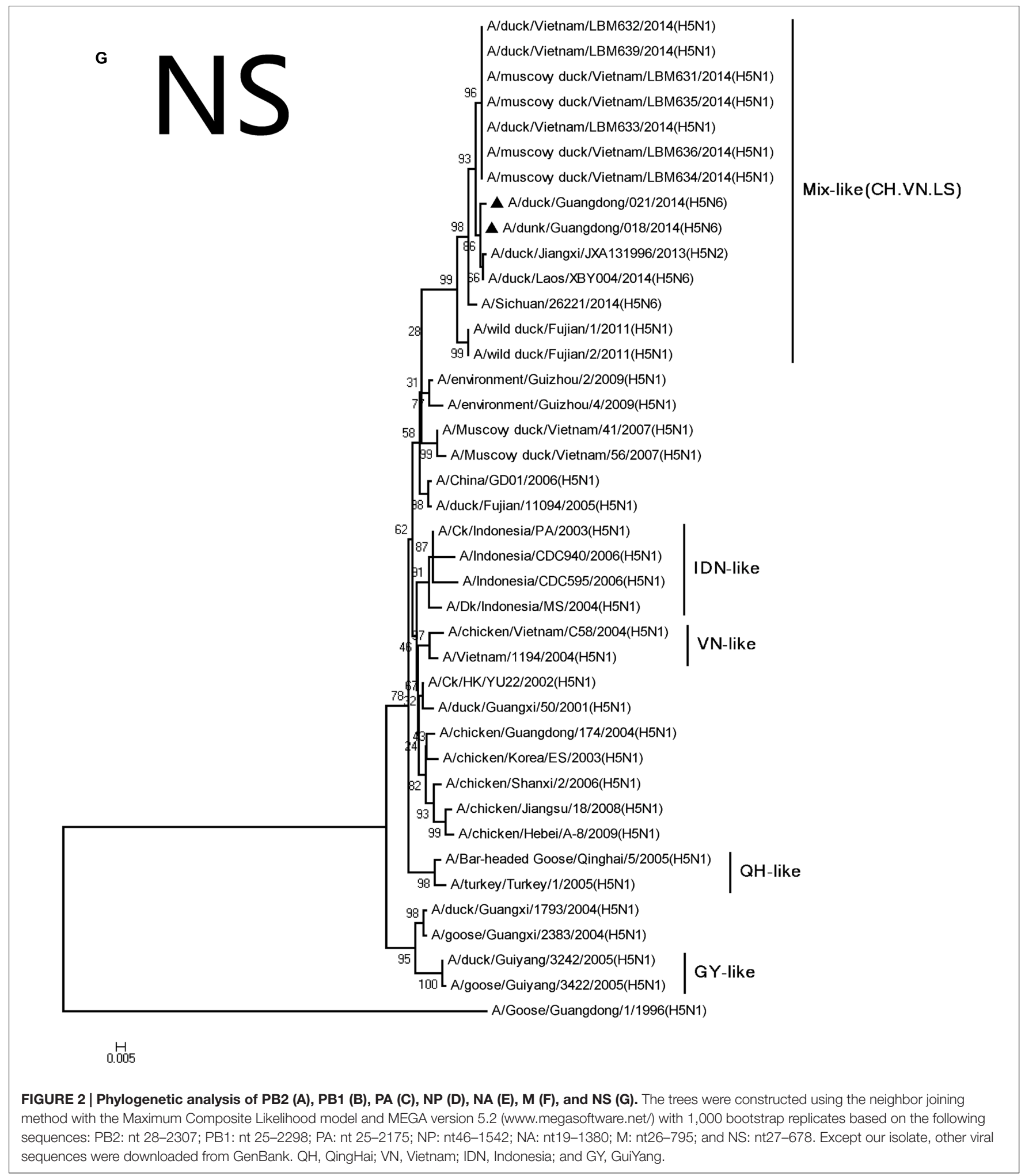

The genes derived from Mix-like viruses included a H5N6 virus-infected human in Sichuan in 2014 (Figure 2). A S31N substitution of the M2 gene was associated with adamantane resistance, but this mutation was not observed in M2.
The NP and NS genes were highly homologous with the viruses A/hill myna/Heilongjiang/0704/2012 (H5N1) and A/wild duck/Fujian/1/2011(H5N1), respectively, (Table 2). The NP and NS genes were derived from the Mix-like viruses 


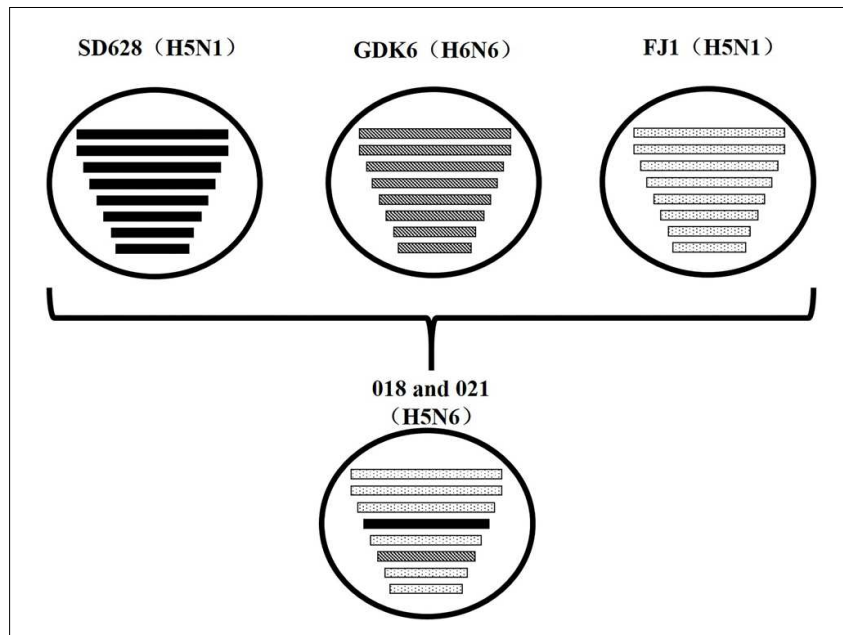

FIGURE 3 | Putative genomic compositions of novel H5N6 influenza viruses isolated from domestic ducks in Southern China 2014 as well as their five possible parent viruses. The eight gene segments (from top to bottom) in each virus were basic polymerase 2 , basic polymerase 1 , acidic polymerase, hemagglutinin, nucleoprotein, neuraminidase, matrix, and non-structural protein. Each pattern represented a separate virus background. Blank indicated SD628 (H5N1); A/wild duck/Shandong/628/2011 (H5N1); bias indicated GDK6 (H6N6), A/swine/Guangdong/K6/2010(H6N6); spot indicated FJ1 (H5N1), AWild duck/Fujian/1/2011(H5N1). The simplified schematic illustration was based on nucleotide-distance comparison and phylogenetic analysis.

that included a H5N6-infected human in Sichuan in 2014 (Figure 2). The 678 amino acid residue of $\mathrm{PB1}$ was $\mathrm{S}$, the 615 amino acid residue of $\mathrm{PA}$ was $\mathrm{K}$, and the 319 amino acid residue of NP was N. This indicated no genetic markers associated with increased virulence in mammals (Gabriel et al., 2005).

\section{DISCUSSION}

Since the late 1990s, multiple genotype H5N1 and H6N6 influenza viruses have been circulating in Chinese poultry populations ( $\mathrm{Li}$ et al., 2004; Wang et al., 2014). This provides abundant gene pools for further inter- or intra-subtype reassortment. In recent years, an increasing number of novel avian viruses have been reported to infect humans. On May 7, 2013, the first human infection with H5N6 AIV was found in a 49-year-old man living in Nanchong City, China. This was confirmed to be the H5N6 virus from the patient's poultry farm. Additional human infections with H5N6 were documented in December 2014. On February 9, 2015, a third human H5N6 infection was reported and was fatal.

In our study, the reassortant influenza viruses [018 (H5N6) and 021 (H5N6)] were isolated from apparently healthy domestic ducks in early March 2014. They were completely lethal in chickens (100\% mortality), and the mean death time (MDT) was 0-7 DPI. The viruses could also cause horizontal transmission in chickens by naïve contact. All the naïve contact chickens in these two groups show the same clinical signs as infected chickens, and the MDT was 6 or 7 DPI. Moreover, the two viruses could replicate systemically in chickens. Virus was found in all the tested organs at high titer levels-especially the lung. The short incubation period and rapid progression of the H5N6 viruses were consistent with HPAI. Thus, our findings showed that the H5N6 viruses from waterfowl were highly pathogenic to chickens and could transmit among them.

Their HA genes were Mix-like and were assigned to clade 2.3.4.4. Their NA genes were derived from H6N6-like Eurasian lineage viruses; the internal genes included M, NS, NP, PA, PB1, and PB2 derived from Mix-like viruses. In addition, the H5N6 virus-infected human in Sichuan in 2014 also belongs to the Mixlike viruses. Such Mix-like gene pools are characterized by H5N1 and H5N6 subtypes of AIVs isolated from Vietnam and Laos. In addition, a small amount of $\mathrm{H} 5 \mathrm{~N} 1$ and $\mathrm{H} 5 \mathrm{~N} 2$ viruses were isolated in China. The H5N1 gene pool has widened and emerged since 2003.

In the future, the community must study whether this Mixlike gene pool will become more diverse and even branch into a new subtype/genotype. Moreover, the H5N6 avian influenza broke out in Laos in late March 2014. The highly homologous nucleotide in our study suggested that our isolates might have an identical origin with the A/duck/Laos/LPQ001/2014(H5N6) virus from Laos. The NA genes of our isolates and the LPQ001 virus were highly homologous with the virus A/swine/Guangdong/K6/2010(H6N6). The other genes of our viruses and the LPQ001 virus were highly homologous with the H5N1 viruses from Fujian, respectively, (Figure 3). Moreover, the data suggested that the HA genes and the internal genes of $\mathrm{H} 5 \mathrm{~N} 1$ viruses from Vietnam are highly related to those from the H5N6 viruses. Therefore, our results suggested that the H5N6 viruses might circulate among Southern China, Vietnam and Laos.

Reassortant among different influenza viruses is considered the main mechanism for the emergence of new viruses. The co-circulation of $\mathrm{H} 5$ and $\mathrm{H} 6$ influenza viruses in domestic ducks contributed to the antigenic and genetic diversity of influenza viruses in Southern China. Zhejiang, Hubei, and Guangdong in particular had an outbreak of H5N6 avian influenza in farms in 2014 followed by Hunan and Jiangsu in 2015 (World Organization for Animal Health, 2015). In addition, the viruses were reported to have infected humans in Sichuan, Guangdong, and Yunnan, China from 2014 to the present. As these viruses caused $100 \%$ mortality in chickens, these viruses should be monitored in poultry. Their risk to human health cannot be denied and should be monitored in future.

\section{AUTHOR CONTRIBUTIONS}

PJ and JC designed this study, performed the experiments and participated in the data collection and analysis. YS, HS, ZZ, QY, SW, NQ, NW, and GO assisted with animal experiment. PJ and JC drafted the manuscript. ML participated in writing the discussion. 


\section{ACKNOWLEDGMENTS}

This work was supported by grants from the National Natural Science Foundation of China (No. U1501212 and No. 31172343), the Program of International Science and Technology Cooperation of China (No. 2013DFA31940), the Natural Science

\section{REFERENCES}

Belser, J. A., Szretter, K. J., Katz, J. M., and Tumpey, T. M. (2009). Use of animal models to understand the pandemic potential of highly pathogenic avian influenza viruses. Adv. Virus Res. 73, 55-97. doi: 10.1016/S0065-3527(09) 73002-7

Gabriel, G., Dauber, B., Wolff, T., Planz, O., Klenk, H. D., and Stech, J. (2005). The viral polymerase mediates adaptation of an avian influenza virus to a mammalian host. Proc. Natl. Acad. Sci. U.S.A. 102, 18590-18595. doi: 10.1073/pnas.0507415102

Gu, M., Zhao, G., Zhao, K., Zhong, L., Huang, J., Wan, H., et al. (2013). Novel variants of clade 2.3.4 highly pathogenic avian influenza A (H5N1) viruses, China. Emerg. Infect. Dis. 19, 2021-2024. doi: 10.3201/eid1912. 130340

Kawaoka, Y., Chambers, T. M., Sladen, W. L., and Webster, R. G. (1988). Is the gene pool of influenza viruses in shorebirds and gulls different from that in wild ducks? Virology 163, 247-250. doi: 10.1016/0042-6822(88) 90260-7

Li, K. S., Guan, Y., Wang, J., Smith, G. J., Xu, K. M., Duan, L., et al. (2004). Genesis of a highly pathogenic and potentially pandemic $\mathrm{H} 5 \mathrm{~N} 1$ influenza virus in eastern Asia. Nature 430, 209-213. doi: 10.1038/nature02746

Mehle, A., and Doudna, J. A. (2008). An inhibitory activity in human cells restricts the function of an avian-like influenza virus polymerase. Cell Host Microbe 4, 111-122. doi: 10.1016/j.chom.2008.06.007

Nobusawa, E., Aoyama, T., Kato, H., Suzuki, Y., Tateno, Y., and Nakajima, K. (1991). Comparison of complete amino acid sequences and receptor-binding properties among 13 serotypes of hemagglutinins of influenza A viruses. Virology 182, 475-485. doi: 10.1016/0042-6822(91)90588-3

Stevens, J., Blixt, O., Tumpey, T. M., Taubenberger, J. K., Paulson, J. C., and Wilson, I. A. (2006). Structure and receptor specificity of the hemagglutinin from an H5N1 influenza virus. Science 312, 404-410. doi: 10.1126/science.1124513
Foundation of Guangdong Province (No. 10251064201000004), the Science and Technology Projects of Guangdong Province (No. 2012B020306003 and 2014A050503061), the Science and Technology Projects of Guangzhou (No. 201300000037 and 2013J4500030), and the Earmarked Fund for Modern AgroIndustry Technology Research System (CARS-42-G09).

Sun, H., Jiao, P., Jia, B., Xu, C., Wei, L., Shan, F., et al. (2011). Pathogenicity in quails and mice of $\mathrm{H} 5 \mathrm{~N} 1$ highly pathogenic avian influenza viruses isolated from ducks. Vet. Microbiol. 152, 258-265. doi: 10.1016/j.vetmic.2011. 05.009

Wang, G., Deng, G., Shi, J., Luo, W., Zhang, G., Zhang, Q., et al. (2014). H6 influenza viruses pose a potential threat to human health. J. Virol. 88, 39533964. doi: 10.1128/JVI.03292-13

Webster, R. G., Bean, W. J., Gorman, O. T., Chambers, T. M., and Kawaoka, Y. (1992). Evolution and ecology of influenza A viruses. Microbiol. Rev. 56, $152-179$.

WHO/OIE/FAO H5N1 Evolution Working Group (2012). Continued evolution of highly pathogenic avian influenza A (H5N1): updated nomenclature. Influenza Other Respir. Viruses 6, 1-5. doi: 10.1111/j.1750-2659.2011.00298.x

World Organization for Animal Health (2015). Available at: http://www.oie.int/en /animal-health-in-the-world/update-on-avian-influenza/2015/

Yen, H. L., Aldridge, J. R., Boon, A. C., Ilyushina, N. A., Salomon, R., HulsePost, D. J., et al. (2009). Changes in H5N1 influenza virus hemagglutinin receptor binding domain affect systemic spread. Proc. Natl. Acad. Sci. U.S.A. 106, 286-291. doi: 10.1073/pnas.0811052106

Conflict of Interest Statement: The authors declare that the research was conducted in the absence of any commercial or financial relationships that could be construed as a potential conflict of interest.

Copyright (C) 2016 Jiao, Cui, Song, Song, Zhao, Wu, Qu, Wang, Ouyang and Liao. This is an open-access article distributed under the terms of the Creative Commons Attribution License (CC BY). The use, distribution or reproduction in other forums is permitted, provided the original author(s) or licensor are credited and that the original publication in this journal is cited, in accordance with accepted academic practice. No use, distribution or reproduction is permitted which does not comply with these terms. 